\title{
Universal algebraic relaxation of velocity and phase in pulled fronts generating periodic or chaotic states
}

\author{
Cornelis Storm, ${ }^{1}$ Willem Spruijt, ${ }^{1}$ Ute Ebert,,${ }^{1,2}$ and Wim van Saarloos ${ }^{1}$ \\ ${ }^{1}$ Instituut-Lorentz, Universiteit Leiden, Postbus 9506, 2300 RA Leiden, The Netherlands \\ ${ }^{2}$ Centrum voor Wiskunde en Informatica, Postbus 94079, 1090 GB Amsterdam, The Netherlands
}

(Received 23 December 1999)

\begin{abstract}
We investigate the asymptotic relaxation of so-called pulled fronts propagating into an unstable state, and generalize the universal algebraic velocity relaxation of uniformly translating fronts to fronts that generate periodic or even chaotic states. A surprising feature is that such fronts also exhibit a universal algebraic phase relaxation. For fronts that generate a periodic state, like those in the Swift-Hohenberg equation or in a Rayleigh-Bénard experiment, this implies an algebraically slow relaxation of the pattern wavelength just behind the front, which should be experimentally testable.
\end{abstract}

PACS number(s): 05.45.-a, 47.54.+r, 47.20.Ky, 02.30.Jr

Many systems, when driven sufficiently far from equilibrium, spontaneously organize themselves in coherent or incoherent patterns [1]. While the "selection" of a final state pattern can be determined by a variety of dynamical mechanisms, or even the competition thereof, the final state selection by a propagating "pulled" front turns out to be remarkably simple and robust. So-called pulled fronts propagate into a linearly unstable state and are almost literally being "pulled along" by the leading edge of the profile whose dynamics is governed by the linearization about the unstable state [2-5]: Their asymptotic speed is equal to the linear spreading speed $v^{*}$ of linear perturbations about the unstable state.

Recently, it was discovered that non-pattern-generating pulled fronts, which asymptotically are uniformly translating, relax to their asymptotic velocity and shape very slowly with a power law. This relaxation is in fact remarkably universal. However, the clearest and most relevant examples, Taylor vortex fronts [6], fronts in Rayleigh-Bénard cells [7] or, in the pearling instability [8], are all pattern forming: these fronts leave a (nearly) periodic pattern behind. From this perspective, the main result we derive in this Rapid Communication has both conceptual and practical implications: We show that the results for the velocity relaxation derived in [5] not only extend to pattern forming and chaotic fronts, but that in addition there is a similar power law relaxation of the wavelength just behind a coherent pattern forming front. This latter relaxation appears more easily accessible experimentally than that of the front velocity.

Our results can be summarized as follows: pattern forming or chaotic pulled fronts emerging from "steep" initial conditions (i.e., falling off faster than $e^{-\lambda^{*} x}$ for $x \rightarrow \infty$ ), have a universal power law relaxation of their velocity $v(t)$ and phase $\Gamma(t)$ with time $t$,

$$
\begin{gathered}
v(t) \equiv v^{*}+\dot{X}(t), \\
\dot{X}(t)=-\frac{3}{2 \lambda^{*} t}+\frac{3 \sqrt{\pi}}{2 \lambda^{* 2} t^{3 / 2}} \operatorname{Re}\left(\frac{1}{\sqrt{D}}\right)+\mathcal{O}\left(\frac{1}{t^{2}}\right),
\end{gathered}
$$

$$
\dot{\Gamma}(t)=-q^{*} \dot{X}(t)-\frac{3 \sqrt{\pi}}{2 \lambda * t^{3 / 2}} \operatorname{Im}\left(\frac{1}{\sqrt{D}}\right)+\mathcal{O}\left(\frac{1}{t^{2}}\right) .
$$

As explained below, the coefficients $v^{*}, k^{*}=q^{*}+i \lambda^{*}$, and $D$ are all given explicitly in terms of the dispersion relation of the linearized equation. As we shall see, $X(t)$ has the meaning of a collective coordinate of the front in the frame moving with the asymptotic velocity $v^{*}$. For a front that generates a coherent (almost) periodic pattern, our results imply that the local wavelength $\Lambda$ just behind the front, where the envelope begins to saturate, also relaxes as $1 / t$ to its asymptotic value: it is given by

$$
\Lambda(t)=2 \pi\left|\frac{v^{*}+\dot{X}(t)}{\Omega^{*}+\dot{\Gamma}(t)}\right|+\mathcal{O}\left(\frac{1}{t^{2}}\right)
$$

with the frequency $\Omega^{*}$ also given below. As $\dot{X}(t)$ and $\dot{\Gamma}(t)$ are explicitly given by Eqs. (2) and (3), this immediately yields $\Lambda(t)$ up to order $t^{-3 / 2}$ in time.

Before summarizing our derivation, we explain what we mean by velocity and phase for the various types of fronts.

Uniformly translating pulled fronts. The simplest types of fronts are those for which the dynamical field $\phi(x, t)$ asymptotically approaches a uniformly translating profile $\phi$ $\equiv \Phi_{v^{*}}(\xi), \xi=x-v^{*} t$, as happens, e.g., in the celebrated nonlinear diffusion equation $\partial_{t} \phi=\partial_{x}^{2} \phi+\phi-\phi^{3}$ for fronts propagating into the unstable $\phi=0$ state. If we define level curves as the lines in an $x, t$ diagram where $\phi(x, t)$ has a particular value, we can define the velocity $v(t)$ as the slope of a level curve. For uniformly translating fronts, $q^{*}=0$ $=\operatorname{Im} D$; Eq. (2) then reduces to the expression derived for uniformly translating fronts in [5]. The remarkable point is that the expression for $v(t)$ is in this case completely independent of which level curve one traces. Moreover, it was shown in [5] that the nonlinear front region is slaved to the leading edge of the front whose velocity relaxes according to Eq. (2). This results in

$$
\phi(x, t)=\Phi_{v(t)}\left(\xi_{X}\right)+\mathcal{O}\left(t^{-2}\right), \quad \xi_{X} \ll \sqrt{t},
$$



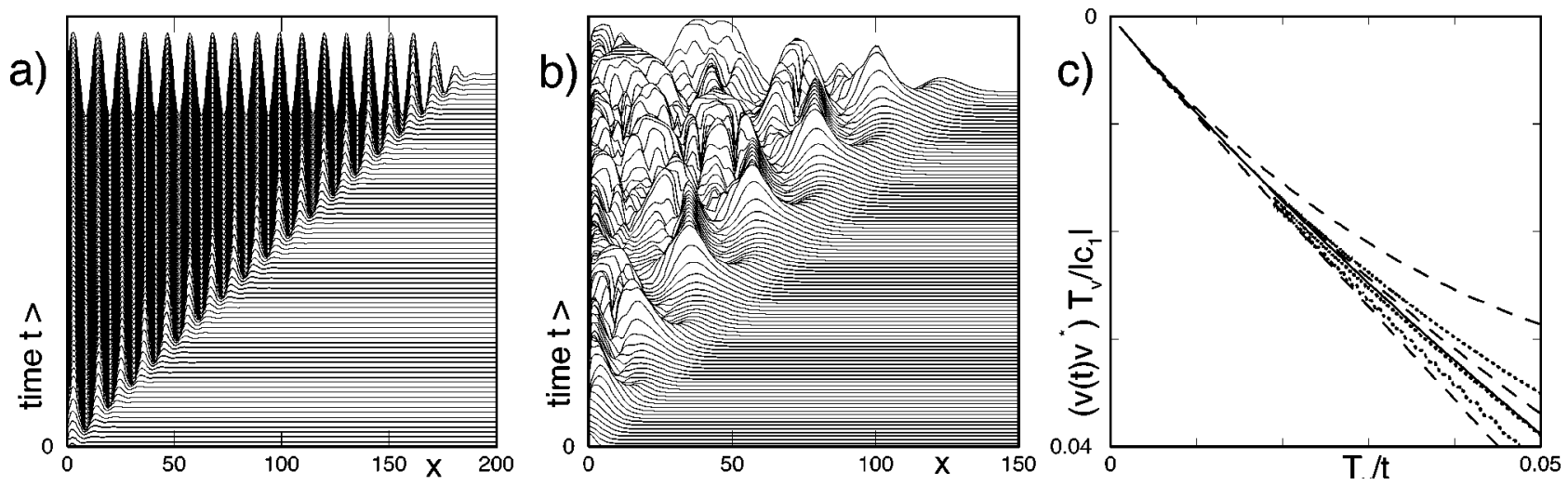

FIG. 1. (a) Space-time plot of a pulled front in the SH Eq. (7) with $\varepsilon=5$ and Gaussian initial conditions. Time steps between successive lines are 0.1. (b) A pulled front in the QCGL Eq. (9) with $\varepsilon=0.25, C_{1}=1, C_{3}=C_{5}=-3$, and Gaussian initial conditions. Plotted is $|A(x, t)|$. Time steps between lines are 1. (c) Scaling plot of the velocity relaxation $\left[v(t)-v^{*}\right] \cdot T_{v} /\left|c_{1}\right|$ vs $1 / \tau$ with $\tau=t / T_{v}$ and characteristic time $T_{v}=\left(c_{3 / 2} / c_{1}\right)^{2}$. Plotted are, from left to right, the data for the SH equation for heights $u=\sqrt{\varepsilon}, 0.01 \sqrt{\varepsilon}$, and $0.0001 \sqrt{\varepsilon}(\varepsilon=5)$ as dashed lines, and for the QCGL Eq. (9) for heights $|A|=0.002,0.0002$, and 0.00002 as dotted lines. The solid line is the universal asymptote $-1 / \tau+1 / \tau^{3 / 2}$

$$
\xi_{X}=x-v^{*} t-X(t)
$$

where $\Phi_{v}(\xi), \xi=x-v t$ solves the ordinary differential equation (ODE) for a front propagating uniformly with velocity $v . v(t)$ in Eq. (5) is the instantaneous velocity of the front, and the frame $\xi_{X}$ is shifted by the time dependent quantity $X(t)$. Since the collective coordinate $X(t)$ diverges as $\ln t$ for large $t$ according to Eq. (2), the difference between $\xi_{X}$ and a uniformly translating frame is crucial; only in the former can we follow the relaxation. Uniformly translating fronts have no phase, hence all terms in Eq. (3) vanish identically.

Coherent pattern generating fronts. As an example of coherent pattern generating fronts, we consider the so-called Swift-Hohenberg $(\mathrm{SH})$ equation

$$
\partial_{t} u=\varepsilon u-\left(1+\partial_{x}^{2}\right)^{2} u-u^{3}, \quad \varepsilon>0 .
$$

The space-time plot of Fig. 1(a) illustrates how SH fronts with steep initial conditions generate a periodic pattern. It is known that they are pulled $[2,4,9]$. In this case, new level curves in an $x, t$ plot are constantly being generated. If we define in this case the velocity as the slope of the uppermost level curve, one gets an oscillatory function. Its average is $v(t)$ given in Eq. (1), but $v(t)$ is difficult to extract this way. Numerically, it is better to determine the velocity from an empirical envelope obtained by interpolating the positions of the maxima. Since these pattern forming front solutions for long times have a temporal periodicity $u(\xi, t)=u(\xi, t+T)$ in the frame $\xi=x-v t$ moving with the velocity $v$ of the front, the asymptotic profiles can be written in the form $\sum_{n=1} e^{-2 \pi i n t / T} U_{v}^{n}(\xi)+$ c.c. In terms of these complex modes $U$, our result for the relaxation of the interior region of the pulled front becomes in analogy to Eq. (5)

$$
u(x, t) \simeq \sum_{n=1} e^{-n i \Omega^{*} t-n i \Gamma(t)} U_{v(t)}^{n}\left(\xi_{X}\right)+\text { c.c. }+\cdots,
$$

with the frequency $\Omega *$ given below. Equation (8) shows that $\Gamma(t)$ is the global phase of the relaxing profile, as the functions $U_{v}^{n}$ only have a $\xi_{X}$ dependence. The result of our cal- culation of the long time relaxation of $v(t)$ and $\Gamma(t)$ is given in Eqs. (1)-(3). In principle, the relaxation of the local wavelength behind the front depends both on $\dot{\Gamma}(t)$ and on the phase difference of $U_{v(t)}^{n}$ before and behind the front implied by the $v(t)$ dependence. However, this phase relaxation is proportional to $v(t)-v^{*}$ and hence is of lower order than the two leading terms of $\Gamma(t)$. To order $t^{-3 / 2}$, Eq. (4) then immediately follows from Eq. (8). This can be viewed as a generalization of an earlier argument $[2,4]$ using the conservation of nodes.

Incoherent or chaotic fronts. The third class we consider consists of fronts which leave behind chaotic states. They occur in some regions of parameter space in the cubic complex Ginzburg-Landau equation [10] or in the quintic extension (QCGL) [11] that we consider here,

$$
\begin{aligned}
\partial_{t} A= & \varepsilon A+\left(1+i C_{1}\right) \partial_{x}^{2} A+\left(1+i C_{3}\right)|A|^{2} A \\
& -\left(1-i C_{5}\right)|A|^{4} A .
\end{aligned}
$$

Figure 1(b) shows an example of a pulled front in this equation. Level curves in a space-time diagram can now also both start and end. If we calculate the velocity from the slope of the uppermost level line, then its average value is again given by Eq. (2) [12], but the oscillations can be quite large. However, our analysis confirms what is already visible in Fig. 1(b), namely, that even a chaotic pulled front becomes more coherent the further one looks into the leading edge of the profile. Indeed we will see that in the leading edge where $|A| \ll 1$ the profile is given by an expression reminiscent of Eq. (8),

$$
A(x, t) \approx e^{-i \Omega * t-i \Gamma(t)} e^{i k^{*} \xi_{X}} \psi\left(\xi_{X}\right), \quad 1 \ll \xi_{X} \ll \sqrt{t} .
$$

The fluctuations about this expression become smaller the larger $\xi_{X}$.

In Figs. 1(c) and 2(c) we show as an example results of our simulations of the SH equation (7) and the QCGL (9). They fully confirm our predictions (2) and (3) for the asymptotic average velocity and phase relaxation. 

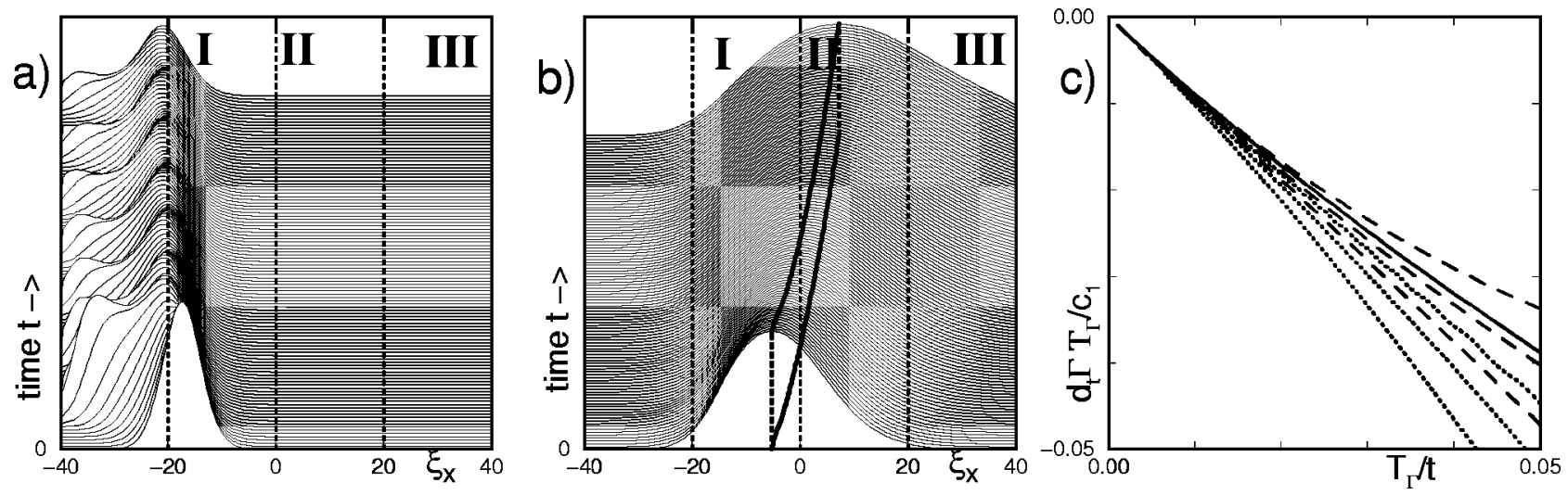

FIG. 2. (a) and (b) Simulation of the QCGL equation as in Fig. 1(b) for times $t=35$ to 144. (a) shows $|N|$ (16) as a function of $\xi_{X}$. (b) shows $|\psi|$, which in region I builds up a linear slope $\psi \propto \alpha \xi_{X}$, and in region III decays like a Gaussian widening in time. The lines in region II show the maxima of $\psi\left(\xi_{X}, t\right)$ for fixed $t$ and their projection $\xi_{X} \sim \sqrt{t}$ into the $\left(\xi_{X}, t\right)$ plane. (c) shows the scaling plot for the phase relaxation. From left to right: SH (dashed lines) for $u=\sqrt{\varepsilon}, 0.01 \sqrt{\varepsilon}$, and $0.0001 \sqrt{\varepsilon}(\varepsilon=5)$, and QCGL (dotted lines) for $|A|=0.002$, 0.0002 , and 0.00002 . Plotted is $\dot{\Gamma}(t) T_{\Gamma} / c_{1}$ vs $1 / \tau$. Here $\tau=t / T_{\Gamma}$, and $T_{\Gamma}=T_{v}\left[1+\lambda^{*} \operatorname{Im} D^{-1 / 2} /\left(q^{*} \operatorname{Re} D^{-1 / 2}\right)\right]$. The solid line again is the universal asymptote $-1 / \tau+1 / \tau^{3 / 2}$.

We now summarize how these results arise for the case of a single (scalar) equation. The extension to the case of coupled equations can be done along the lines of [5].

Calculation of the asymptotic parameters. We first briefly summarize how the linear spreading velocity $v^{*}$ and the associated parameters $\lambda^{*}$ etc. arise $[13,5]$. After linearization about the unstable state, the equations we consider can all be written in the form $\partial_{t} \phi=\mathcal{L}\left(\partial_{x}, \partial_{x}^{2}, \cdots\right) \phi$. For a Fourier mode $e^{-i \omega t+i k x}$, this yields the dispersion relation $\omega(k)$. The linear spreading velocity $v^{*}$ of steep initial conditions is then obtained by a saddle point analysis of the Green's function $G$ of these equations. In the asymptotic frame $\xi=x-v^{*} t$, $G(\xi, t)$ becomes

$$
G(\xi, t)=\int \frac{d k}{2 \pi} e^{-i \Omega(k) t+i k \xi} \approx e^{i k^{*} \xi-i \Omega^{*}} \frac{e^{-\left(\xi^{2} / 4 D t\right)}}{\sqrt{4 \pi D t}}
$$

for large times. Here $\Omega(k)=\omega(k)-v^{*} k$, and

$$
\left.\frac{d \Omega(k)}{d k}\right|_{k^{*}}=0, \quad \operatorname{Im} \Omega\left(k^{*}\right)=0, \quad D=\left.\frac{i d^{2} \Omega(k)}{2 d k^{2}}\right|_{k^{*}} .
$$

The first equation in Eq. (12) is the saddle point condition, while the second one expresses the self-consistency condition that there is no growth in the comoving frame. These equations straightforwardly determine $v^{*}, k^{*}=q^{*}+i \lambda^{*}, D$ and the real frequency $\Omega^{*}=\Omega\left(k^{*}\right)$ [14].

Choosing the proper frame and transformation. Equation (11) confirms that a localized initial condition will grow out and spread asymptotically with the velocity $v^{*}$ given by Eq. (12). Our aim now is to understand the convergence of a pulled front due to the interplay of the linear spreading and the nonlinearities. The Green's function expression (11) gives three important hints in this regard: First of all, $G(\xi, t)$ is asymptotically of the form $e^{i k^{*} \xi-i \Omega^{*} t}$ times a crossover function whose diffusive behavior is betrayed by the Gaussian form in Eq. (11). Hence, if we write our dynamical fields as $A=e^{i k^{*} \xi^{-i \Omega^{*} t}} \psi(\xi, t)$ for the QCGL (9) or $u$ $=e^{i k^{*} \xi-i \Omega^{*} t} \psi(\xi, t)+$ c.c. for the real field $u$ in Eq. (7), we expect that the dynamical equation for $\psi(\xi, t)$ obeys a diffusion-type equation. Second, as we have argued in [5], for the relaxation analysis one wants to work in a frame where the crossover function $\psi$ becomes asymptotically time independent. This is obviously not true in the $\xi$ frame, due to the factor $1 / \sqrt{t}$ in Eq. (11). However, this term can be absorbed in the exponential prefactor, by writing $t^{-\nu} e^{i k^{*} \xi-i \Omega * t}=e^{i k^{*} \xi-i \Omega * t-\nu \ln t}$. Hence, we introduce the logarithmically shifted frame $\xi_{X}=\xi-X(t)$ [5], as already used in Eq. (6). Third, we find a feature specific for pattern forming fronts: the complex parameters, and $D$ in particular, lead us to introduce the global phase $\Gamma(t)$. We expand $\Gamma(t)$ like $\dot{X}(t)[5]$,

$$
\dot{X}(t)=\frac{c_{1}}{t}+\frac{c_{3 / 2}}{t^{3 / 2}}+\cdots, \quad \dot{\Gamma}(t)=\frac{d_{1}}{t}+\frac{d_{3 / 2}}{t^{3 / 2}}+\cdots
$$

and analyze the long time dynamics by performing a "leading edge transformation", to the field $\psi$,

$$
\begin{aligned}
& \text { QCGL: } \quad A=e^{i k^{*} \xi_{X}-i \Omega * t-i \Gamma(t)} \psi\left(\xi_{X}, t\right), \\
& \text { SH: } \quad u=e^{i k^{*} \xi_{X}-i \Omega * t-i \Gamma(t)} \psi\left(\xi_{X}, t\right)+\text { c.c. }
\end{aligned}
$$

Steep initial conditions imply that $\psi\left(\xi_{X}, t\right) \rightarrow 0$ as $\xi_{X} \rightarrow \infty$. The determination of the coefficients in the expansions (13) of $\dot{X}$ and $\dot{\Gamma}$ is the main goal of the subsequent analysis, as this then directly yields Eqs. (2) and (3).

Understanding the intermediate asymptotics. Substituting the leading edge transformation (14) into the nonlinear dynamical equations, we get

$$
\begin{aligned}
\partial_{t} \psi= & D \partial_{\xi_{X}}^{2} \psi+\sum_{n=3} D_{n} \partial_{\xi_{X}}^{n} \psi \\
& +\left[\dot{X}(t)\left(\partial_{\xi_{X}}+i k^{*}\right)+i \dot{\Gamma}(t)\right] \psi-N(\psi),
\end{aligned}
$$


with $D_{n}=(-i / n !) d^{n} \Omega /\left.(d i k)^{n}\right|_{k *}$ the generalization of $D$ in Eq. (12) [of course, for the QCGL, $\Omega(k)$ is quadratic in $k$, so $\left.D_{n}=0\right]$. In this equation, $N$ accounts for the nonlinear terms; e.g., for the QCGL, we simply have

$$
N=e^{-2 \lambda * \xi_{X}}|\psi|^{2} \psi\left[1-i C_{3}+\left(1-i C_{5}\right) e^{-2 \lambda * \xi_{X}}|\psi|^{2}\right] .
$$

The expression for the $\mathrm{SH}$ equation is similar.

The structure of Eq. (15) is that of a diffusion-type equation with $1 / t$ and higher order corrections from the $\dot{X}$ and $\dot{\Gamma}$ terms, and with a nonlinearity $N$. The crucial point to recognize now is that for fronts, $N$ is nonzero only in a region of finite width: For $\xi_{X} \rightarrow \infty, N$ decays exponentially due to the explicit exponential factors in Eq. (16). For $\xi_{X} \rightarrow-\infty, N$ also decays exponentially, since $u$ and $A$ remain finite, so that $\psi$ decays as $e^{-\lambda *\left|\xi_{X}\right|}$ according to Eq. (14). Intuitively, therefore, we can think of Eq. (15) as a diffusion equation in the presence of a sink $N$ localized at some finite value of $\xi_{X}$. The ensuing dynamics of $\psi$ to the right of the sink can be understood with the aid of Figs. 2(a) and 2(b), which are obtained directly from the time-dependent numerical simulations of the QCGL (9). To extract the intermediate asymptotic behavior illustrated by these plots, we integrate Eq. (15) once to get

$$
\begin{aligned}
\partial_{t} \int_{-\infty}^{\xi_{X}} d \xi_{X}^{\prime} \psi= & D \partial_{\xi_{X}} \psi+\sum_{n=3} \frac{D_{n}}{n-1} \partial_{\xi_{X}}^{n-1} \psi \\
& +i\left[k^{*} \dot{X}(t)+\dot{\Gamma}(t)\right] \int_{-\infty}^{\xi_{X}} d \xi_{X}^{\prime} \psi+\dot{X}(t) \psi \\
& -\int_{-\infty}^{\xi_{X}} d \xi_{X}^{\prime} N(\psi)
\end{aligned}
$$

Now, in the region labeled I in Fig. 2(b), we have for fixed $\xi_{X}$ and $t \rightarrow \infty$ that the terms proportional to $\dot{X}$ and $\dot{\Gamma}$ can be neglected upon averaging over the fast fluctuations; the same holds for the term on the left. Since the integral converges quickly to the right due to the exponential factors in $N$, we then get immediately, irrespective of the presence of higher order spatial derivatives

$$
\lim _{t \rightarrow \infty} D \frac{\overline{\partial \psi}}{\partial \xi_{X}}=\int_{-\infty}^{\infty} d \xi_{X} \overline{N(\psi)} \equiv \alpha D
$$

Here, the overbar denotes a time average (necessary for the case of a chaotic front). Thus, for large times in region $\mathrm{I}, \bar{\psi}$ $\approx \alpha \xi_{X}+\beta$ in dominant order. Moreover, from the diffusive nature of the equation, our assertion that the fluctuations of $\psi$ rapidly decrease to the right of the region where $N$ is nonzero comes out naturally. In other words, provided that the timeaveraged sink strength $\alpha$ is nonzero, $\alpha \neq 0$, one will find a buildup of a linear gradient in $|\bar{\psi}|$ in region I, independent of the precise form of the nonlinearities or of whether or not the front dynamics is coherent. This behavior is clearly visible in Fig. 2(b). We can understand the dynamics in regions II and III along similar lines. In region III the dominant terms in Eq. (15) are the one on the left and the first one on the second line, and the crossover region II which separates regions I and III moves to the right according to the diffusive law $\xi_{X} \sim D \sqrt{t}$.

Systematic expansion. These considerations are fully corroborated by our extension of the analysis of [5]. Anticipating that $\psi$ falls off for $\xi_{X} \gg 1$, we split off a Gaussian factor by writing $\psi\left(\xi_{X}, t\right)=G(z, t) e^{-z}$ in terms of the similarity variable $z=\xi_{X}^{* 2} /(4 D t)$, and expand

$$
G(z, t)=t^{1 / 2} g_{-1 / 2}(z)+g_{0}(z)+t^{-1 / 2} g_{1 / 2}(z)+\cdots .
$$

This, together with the expansion (13) for $X(t)$ and $\Gamma(t)$, the left "boundary condition"' that $\psi\left(\xi_{X}, t \rightarrow \infty\right)=\alpha \xi_{X}+\beta$ and the condition that the functions $g(z)$ do not diverge exponentially, then results in the expressions (2) for $\dot{X}(t)$ and (3) for $\dot{\Gamma}[9]$. For the QCGL, the analysis immediately implies the result (10) for the front profile in the leading edge. In addition for the $\mathrm{SH}$ equation, one arrives at Eq. (8) for the shape relaxation in the front interior along the lines of [5]: Starting from the ODEs for the $U_{v}^{n}$, one finds upon transforming to the frame $\xi_{X}$ that to $\mathcal{O}\left(t^{-2}\right)$, the time dependence only enters parametrically through $v(t)$. This then yields Eq. (8).
[1] M. C. Cross and P. C. Hohenberg, Rev. Mod. Phys. 65, 851 (1993).

[2] G. Dee and J. S. Langer, Phys. Rev. Lett. 50, 383 (1983).

[3] E. Ben-Jacob, H.R. Brand, G. Dee, L. Kramer, and J.S. Langer, Physica D 14, 348 (1985).

[4] W. van Saarloos, Phys. Rev. A 39, 6367 (1989).

[5] U. Ebert and W. van Saarloos, Phys. Rev. Lett. 80, 1650 (1998); Physica D (to be published); e-print cond-mat/0003181.

[6] G. Ahlers and D. S. Cannell, Phys. Rev. Lett. 50, 1583 (1983).

[7] J. Fineberg and V. Steinberg, Phys. Rev. Lett. 58, 1332 (1987).

[8] R. Bar-Ziv and E. Moses, Phys. Rev. Lett. 73, 1392 (1994); T.

R. Powers and R. E. Goldstein, ibid. 78, 2555 (1997).

[9] U. Ebert, W. Spruijt, and W. van Saarloos (unpublished).
[10] K. Nozaki and N. Bekki, Phys. Rev. Lett. 51, 2171 (1983).

[11] W. van Saarloos and P. C. Hohenberg, Physica D 56, 303 (1992).

[12] This is true for chaotic fronts provided that the temporal correlation function for the chaotic variable falls off at least as fast as $t^{-2}$, so that the temporal change of the average velocity $v(t)$ can be considered adiabatically.

[13] E. M. Lifshitz and L.P. Pitaevskii, Physical Kinetics (Pergamon, New York, 1981).

[14] For Eq. (9), $v^{*}=2 \sqrt{\varepsilon\left(1+C_{1}^{2}\right)}, k^{*}=\left(C_{1}+i\right) \sqrt{\varepsilon /\left(1+C_{1}^{2}\right)}$, $\Omega^{*}=-C_{1} \varepsilon$, and $D=\left(1+i C_{1}\right)$. For Eq. (7), $\lambda^{*}=[(\sqrt{1+6 \varepsilon}$ $-1) / 12]^{1 / 2}, \quad q^{*}= \pm \sqrt{1+3 \lambda^{* 2}}, \quad v^{*}=8 \lambda *\left(1+4 \lambda^{* 2}\right), \quad \Omega *$ $=-8 \lambda * q^{* 3}$, and $D=4 q^{* 2}+12 i q^{*} \lambda^{*}$. 